\title{
Author Correction: Drier Climatic Conditions Increase Withanolide Content of Withania coagulans Enhancing its Inhibitory Potential against Human Prostate Cancer Cells
}

\author{
Samiya Rehman ${ }^{1} \cdot$ Ken Keefover-Ring ${ }^{2} \cdot$ Ihsan ul Haq $^{3} \cdot$ Erum Dilshad $^{4}$. \\ Mohammad Imran Khan ${ }^{5}$ - Nosheen Akhtar ${ }^{6}$. Bushra Mirza ${ }^{1}$ \\ Published online: 10 January 2019 \\ (C) Springer Science+Business Media, LLC, part of Springer Nature 2019
}

\section{Author Correction: Applied Biochemistry and Biotechnology https://doi.org/10.1007/s12010-018-02933-8}

The original version of this article unfortunately contained a mistake in the image of Figure 1 and in Table 4. The corrected version of the figure and table is shown here.

The online version of the original article can be found at https://doi.org/10.1007/s12010-018-02933-8

Samiya Rehman

samiyarehmans@gmail.com

1 Department of Biochemistry, Quaid-i-Azam University, Islamabad, Pakistan

2 Departments of Botany and Geography, University of Wisconsin, Madison, Madison, WI 53706, USA

3 Department of Pharmacy, Quaid-i-Azam University, Islamabad, Pakistan

4 Department of Bioinformatics and Biosciences, Capital University of Science and Technology, Islamabad, Pakistan

5 Department of Biochemistry, King Abdulaziz University, Jeddah, Saudi Arabia

6 National University of Medical Sciences, Rawalpindi, Pakistan 


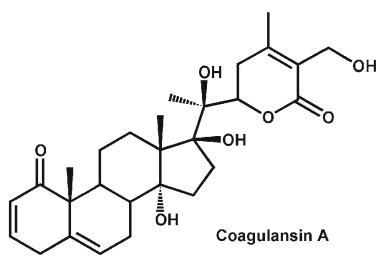<smiles>CC1=C(CO)C(=O)OC([C@](C)(O)[C@H]2CCC3=C4CC=C5CC=CC(=O)[C@]5(C)C4CC[C@@]32C)C1</smiles><smiles>CC1=C(CO)C(=O)OC([C@](C)(O)[C@H]2CC=C3C4CC=C5CC=CC(=O)[C@]5(C)C4CC[C@]32C)C1</smiles><smiles>CC1=C(C)C(=O)OC([C@](C)(O)C2CCC3(O)C4CC=C5CC=CC(=O)[C@]5(C)C4CC[C@]23C)C1</smiles><smiles>CC1=C(CO)C(=O)OC([C@](C)(O)[C@]2(O)CCC3C4CC=C5CC=CC(=O)[C@]5(C)C4CC[C@]32C)C1</smiles><smiles>CC1=C(CO)C(=O)OC([C@](C)(O)[C@]2(O)CC=C3C4CC=C5CC=CC(=O)[C@]5(C)C4CC[C@]32C)C1</smiles><smiles>CC1=C(C)C(=O)OC([C@](C)(O)[C@]2(O)CC[C@]3(O)C4CC=C5C=CCC(=O)[C@]5(C)C4CC[C@]32C)C1</smiles><smiles>CC1=C(C)C(=O)OC([C@](C)(O)[C@]2(O)CC[C@]3(O)C4CC=C5CC=CC(=O)C5(C)C4CC[C@]32C)C1</smiles>

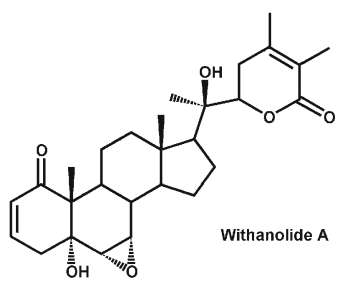<smiles></smiles><smiles>CC1=C(C)C(=O)OC([C@](C)(O)C2CCC3C4CC5O[C@]56C(=O)C=C[C@H](O)C6(C)C4CC[C@@]32C)C1</smiles><smiles></smiles>

Fig. 1 Structures of withanolides found in either plant tissues or leaf extracts and other compounds (withanolide $\mathrm{A}, \mathrm{B}, \mathrm{D}$, and E) with the same exact masses as unknowns 1-5 from the ML and AL leaf extracts 
Table 4 UHPLC-MS retention times, exact masses, and masses of both the deprotonated [(M-H)-] and formate adducts $[(\mathrm{M}-\mathrm{H}+\mathrm{HCOOH})-]$ of available standards and related withanolides

\begin{tabular}{llccc}
\hline Compound & RT $(\mathrm{min})$ & Exact mass & $(\mathrm{M}-\mathrm{H})-$ & $(\mathrm{M}-\mathrm{H}+\mathrm{HCOOH})-$ \\
\hline Available standards & & & & \\
Coagulansin A & 9.70 & 486.2618 & 485.2545 & 531.2600 \\
Withanolide H & 10.69 & 452.2563 & 451.2490 & 497.2545 \\
Withacoagulin G & 13.06 & 470.2668 & 469.2596 & 515.2650 \\
Withacoagulin I & 12.53 & 470.2668 & 469.2596 & 515.2650 \\
Withacoagulin H & 12.03 & 468.2512 & 467.2439 & 513.2494 \\
Withanolide F & 15.48 & 470.2668 & 469.2596 & 515.2650 \\
Withacoagulin & 16.73 & 452.2563 & 451.2490 & 497.2545 \\
Other withanolides with the & $\mathrm{same} \mathrm{exact} \mathrm{masses} \mathrm{as} \mathrm{the} \mathrm{leaf} \mathrm{extract} \mathrm{unknowns}$ & \\
Withanolide A & $\mathrm{Na}$ & 470.2668 & 469.2596 & 515.2650 \\
Withanolide B & $\mathrm{Na}$ & 454.2719 & 453.2646 & 499.2701 \\
Withanolide D & $\mathrm{Na}$ & 470.2668 & 469.2596 & 515.2650 \\
Withanolide E & $\mathrm{Na}$ & 486.2618 & 485.2545 & 531.2600 \\
\hline
\end{tabular}

The author apologizes for this oversight and for any confusion it may have caused.

Publisher's Note Springer Nature remains neutral with regard to jurisdictional claims in published maps and institutional affiliations. 